\title{
Influence of air-powder polishing on bond strength and surface-free energy of universal adhesive systems
}

\author{
Yukie TAMURA, Toshiki TAKAMIZAWA, Yutaka SHIMAMURA, Shunsuke AKIBA, Chiaki YABUKI, Arisa IMAI, \\ Akimasa TSUJIMOTO, Hiroyasu KUROKAWA and Masashi MIYAZAKI \\ Department of Operative Dentistry, Nihon University School of Dentistry, 1-8-13, Kanda-Surugadai, Chiyoda-ku, Tokyo 101-8310, Japan \\ Corresponding author, Toshiki TAKAMIZAWA; E-mail: takamizawa.toshiki@nihon-u.ac.jp
}

\begin{abstract}
The influences of air-powder polishing with glycine or sodium bicarbonate powders on shear bond strengths (SBS) and surface-free energies of universal adhesives were examined. Scotchbond Universal Adhesive (SU, 3M ESPE), G-Premio Bond (GP, GC), Adhese Universal (AU, Ivoclar Vivadent), and All-Bond Universal (AB, Bisco) were used in this study. Bovine dentin surfaces were air polished with glycine or sodium bicarbonate powders prior to the bonding procedure, and resin pastes were bonded to the dentin surface using universal adhesives. SBSs were determined after 24 -h storage in distilled water at $37^{\circ} \mathrm{C}$. Surface-free energy was then determined by measuring contact angles using three test liquids on dentin surfaces. Significantly lower SBSs were observed for dentin that was air-powder polished and surface-free energies were concomitantly lowered. This study indicated that air-powder polishing influences SBSs and surface-free energies. However, glycine powder produced smaller changes in these surface parameters than sodium bicarbonate.
\end{abstract}

Keywords: Air-powder polishing, Universal adhesive, Dentin bond strength, Surface-free energy

\section{INTRODUCTION}

Air-powder polishing is widely used to remove deposits from tooth surfaces and offers a convenient alternative to conventional methods using rubber cups, brush cones, and hand instruments ${ }^{1}$. Moreover, as a contemporary approach, air-powder polishing can provide safe and efficient biofilm and stain removal ${ }^{2}$. Air-powder polishing is superior to rubber-cup polishing when preparing occlusal surfaces prior to etching for sealants ${ }^{3}$. In addition, air-powder polishing of wedgeshaped lesions may enhance dentin bond strengths of resin composites ${ }^{4}$. Previous studies show that air-powder polishing with sodium bicarbonate powder is safe on enamel and is less abrasive than rubber-cup polishing.5). However, decreased bond strengths with dentin were reported following air-powder polishing with sodium bicarbonate powder ${ }^{6)}$.

Glycine powders were recently developed for airpowder polishing, and satisfactory clinical outcomes have been demonstrated in comparison with sodium bicarbonate powder ${ }^{7}$. Accordingly, glycine particles have lower Morse hardness indexes than dentin and have high water solubility and a non-salty taste ${ }^{8}$. In addition, glycine powders reportedly produced less surface damage on restorative materials than sodium bicarbonate powder ${ }^{9}$. However, little is known about adhesion to dentin following abrasion with air-powder polishing using glycine powder.

Dental adhesives with combined single-step functions of self-etching primers and bonding agents are increasingly popular in adhesive restorative dentistry ${ }^{10}$. Recently, multi-mode universal adhesives were introduced to reduce technical sensitivity and to shorten clinical procedure times and can be used as self-etch and etch-and-rinse adhesives ${ }^{11}$. Dentin bond strengths are influenced by smear layers that are created by cutting with either hand or rotary instruments ${ }^{12)}$. Hence, cleaning of dentin surfaces to remove smear layers is a crucial clinical step prior to bonding of restoratives ${ }^{13)}$. Various techniques have been used to modify dentin surfaces and ensure stable bonding of universal adhesives ${ }^{14)}$. Previous studies have shown that sodium bicarbonate air-powder polishing adversely affects dentin bonding of two-step and single-step self-etching adhesives. However, few studies have assessed the influence of air-powder polishing on dentin bond strengths of universal adhesives.

In the present study, we evaluated the influence of air-powder polishing on dentin bond strengths of various universal adhesive systems according to shear bond strengths (SBS) and surface-free energies of air-powder polished dentin surfaces. Specifically, we assessed differences in bond strength and surface-free energy with and without air-powder polishing.

\section{MATERIALS AND METHODS}

\section{Adhesives used}

The materials used in this study are listed in Table 1, and include the universal adhesives Scotchbond Universal Adhesive (SU, 3M ESPE, St. Paul, MN, USA), G-Premio Bond (GP, GC, Tokyo, Japan), Adhese Universal (AU, Ivoclar Vivadent, Schaan, Lichtenstein), and All-Bond Universal (AB, Bisco Dental Products, Schaumburg, IL, USA). Bond strengths were determined for the resin composite Clearfil AP-X (Kuraray Noritake Dental, Tokyo, Japan).

Resin specimens were cured using a visible light curing unit (Optilux 501, Kerr, Danbury, CT, USA), and 
Table 1 Materials

\begin{tabular}{|c|c|c|c|c|}
\hline & Code & Lot No. & Main Components & Manufacturer \\
\hline \multicolumn{5}{|l|}{ Adhesive } \\
\hline $\begin{array}{l}\text { Scotchbond } \\
\text { Universal Adhesive }\end{array}$ & SU & 584163 & $\begin{array}{l}\text { MDP, HEMA, Bis-GMA, } \\
\text { Vitrebond copolymer, ethanol, } \\
\text { water, initiators, filler, silane }\end{array}$ & $\begin{array}{l}\text { 3M ESPE } \\
\text { (St. Paul, MN, USA) }\end{array}$ \\
\hline G-Premio Bond & GP & 1501211 & $\begin{array}{l}\text { MDP, 4-MET, MEPS, } \\
\text { methacrylate monomer, acetone, } \\
\text { water, initiators, filler }\end{array}$ & $\begin{array}{l}\text { GC } \\
\text { (Tokyo, Japan) }\end{array}$ \\
\hline Adhese Universal & $\mathrm{AU}$ & T08671 & $\begin{array}{l}\text { MDP, HEMA, Bis-GMA, } \\
\text { D3MA, MCAP, ethanol, } \\
\text { water, initiator, filler }\end{array}$ & $\begin{array}{l}\text { Ivoclar Vivadent } \\
\text { (Schaan, Lichtenstein) }\end{array}$ \\
\hline All-Bond Universal & $\mathrm{AB}$ & 1300008729 & $\begin{array}{l}\text { MDP, HEMA, Bis-GMA, } \\
\text { ethanol, water, initiators, } \\
\text { silanated colloidal silica }\end{array}$ & $\begin{array}{l}\text { Bisco } \\
\text { (Schaumburg, IL, USA) }\end{array}$ \\
\hline \multicolumn{5}{|l|}{ Air-polishing powder } \\
\hline Air-Flow Perio & GL & 031503 & Glycine & $\begin{array}{l}\text { Electron Medical Systems } \\
\text { (Nyon, Swissland) }\end{array}$ \\
\hline Air-Flow Classic & SB & 041502 & Sodium bicarbonate & Electron Medical Systems \\
\hline
\end{tabular}

MDP: 10-methacryloyloxydecyl dihydrogen phosphate

HEMA: 2-hydroxyethyl methacrylate

Bis-GMA: 2,2-bis[4-(2-hydroxyl-3-methacryloyloxypropoxy)-phenyl]propane

4-MET: 4-methacryloyloxyethyl trimellitic acid

MEPS: Methacryloyloxyalkyl thiophosphate methylmethacrylate

D3MA: Hydrophobic crosslinking dimethacrylate

MCAP: Methacrylated carboxylic acid polymer

the power density (about $600 \mathrm{~mW} / \mathrm{cm}^{2}$ ) of the unit was checked using a dental radiometer (Model 100, Kerr) before preparation of specimens.

\section{Specimen preparation}

Mandibular incisors were extracted from two-threeyear-old cattle and were stored frozen for up to two weeks prior to use as models of human teeth. After removing the roots using a low-speed saw with a diamond-impregnated disk (IsoMet, Buehler, Lake Bluff, IL, USA), pulps were removed, and pulp chambers of each tooth were filled with cotton to avoid penetration of the embedding media. Subsequently, labial surfaces were ground on wet 240-grit silicon carbide ( $\mathrm{SiC}$ ) paper to create flat dentins. Teeth were then mounted in selfcuring acrylic resin (Tray Resin II, Shofu, Kyoto, Japan), with flattened areas exposed, and were then placed under tap water to reduce the temperature rises caused by exothermic polymerization reactions of acrylic resin. Dentin surfaces were finished by grinding with wet 600-grit SiC paper, and were then dried with oil-free compressed air to remove visible water.

\section{Bond strength measurements}

Teeth were divided into three dentin treatment groups, and control specimens were bonded without air-powder polishing prior to applying adhesive. Test specimens were air-powder polished with glycine powder (GL, AirFlow Perio, Electron Medical Systems, Nyon, Swissland) or sodium bicarbonate powder (SB, Air-Flow Classic, Electron Medical Systems). In these procedures, the tip of the air-polishing device (Air-Flow Master, Electron Medical Systems) was positioned approximately $5 \mathrm{~mm}$ above the tooth surface and polishing was performed for $10 \mathrm{~s}$. Dentin surfaces were then rinsed for $10 \mathrm{~s}$ and gently air dried, and adhesives were applied according to respective manufacturers' instructions (Table 2). Subsequently, specimens were clamped in an Ultradent Bonding Jig (Ultradent Products, South Jordan, UT, USA) and resin composites were formed and held on dentin surfaces using plastic molds of 2.4 $\mathrm{mm}$ in internal diameter and $2.0 \mathrm{~mm}$ in height. Resin composites were condensed in molds and were cured for $20 \mathrm{~s}$, and finished specimens were submerged in distilled water at $37^{\circ} \mathrm{C}$ for $24 \mathrm{~h}$.

Ten specimens per group were tested in shear mode using a bond-testing notched-blade semi-circular apparatus (Ultradent Products) in a universal testing machine (Type 5500R, Instron, Norwood, MA, USA) at a crosshead speed of $1.0 \mathrm{~mm} / \mathrm{min}$. SBS values $(\mathrm{MPa})$ were calculated as peak loads at failure divided by specimen surface areas. 
Table 2 Application protocols of adhesives

\begin{tabular}{cl}
\hline Code & \multicolumn{1}{c}{ Application protocol } \\
\hline SU & $\begin{array}{l}\text { Apply adhesive to tooth surface for } 20 \mathrm{~s} \text {. Subject to gentle stream air for } 5 \mathrm{~s} \text { until it no longer moves } \\
\text { and the solvent has evaporated completely. Light irradiation for } 10 \mathrm{~s} .\end{array}$ \\
GP & $\begin{array}{l}\text { Apply adhesive to tooth surface for } 10 \mathrm{~s} \text {. Subject to maximum stream air for } 5 \mathrm{~s} . \\
\text { Light irradiation for } 10 \mathrm{~s} .\end{array}$ \\
AU & $\begin{array}{l}\text { Apply adhesive to tooth surface for } 20 \mathrm{~s} \text { using continuous scrubbing. } \\
\text { Subject to air until a glossy, immobile film layer develops. Light irradiation for } 10 \mathrm{~s} .\end{array}$ \\
& $\begin{array}{l}\text { Rinse preparation and remove excess water. Apply two separate coats of adhesive, } \\
\text { scrubbing the preparation with a micro-brush for } 10-15 \text { s per coat. Air dry for at least } 10 \mathrm{~s} \text { until glossy. } \\
\text { Repeat application of adhesive and air drying. Light irradiation for } 10 \mathrm{~s} .\end{array}$ \\
\hline
\end{tabular}

Table 3 Surface-free energy and component values of test liquids

\begin{tabular}{ccccccc}
\hline Liquid & Lot No. & Manufacturer & $\gamma_{\mathrm{L}}$ & $\gamma_{\mathrm{L}}^{\mathrm{d}}$ & $\gamma_{\mathrm{L}}^{\mathrm{p}}$ & $\gamma_{\mathrm{L}}^{\mathrm{h}}$ \\
\hline 1-bromonaphthalene & ALH4513 & $\begin{array}{l}\text { Wako Pure Chemical } \\
\text { Industries }\end{array}$ & 44.8 & 44.6 & 0.2 \\
Diiodomethane & ALL2310 & $\begin{array}{l}\text { Wako Pure Chemical } \\
\text { Industries }\end{array}$ & 50.8 & 46.8 & 4.0 \\
Distilled water & - & - & 72.8 & 29.1 & 1.3 & 42.4 \\
\hline
\end{tabular}

Unit, $\mathrm{mN} / \mathrm{m}$

$\gamma_{\mathrm{L}}$ : surface-free energy, $\gamma_{\mathrm{L}}^{\mathrm{d}}$ : dispersion force, $\gamma_{\mathrm{L}}^{\mathrm{p}}$ : polarity force, $\gamma_{\mathrm{L}}^{\mathrm{h}}$ : hydrogen bonding force

(a)

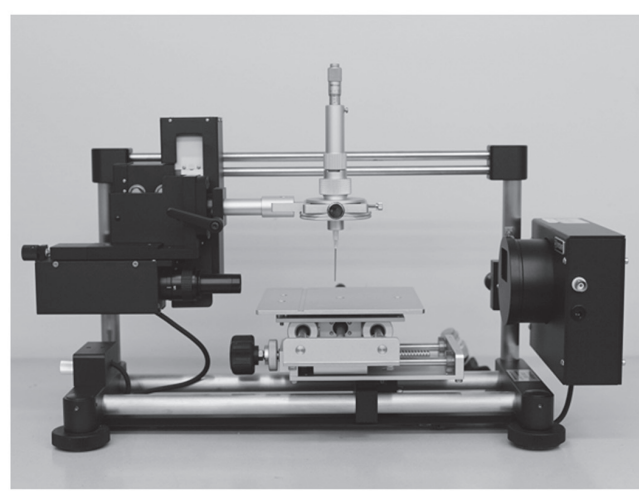

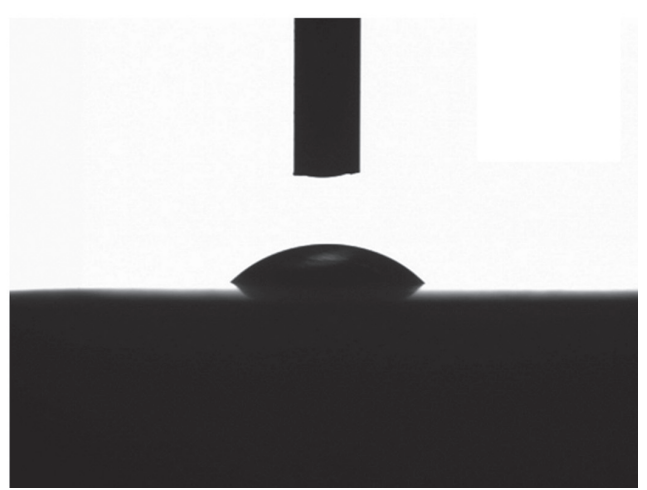

Fig. 1 Drop Master DM500 apparatus fitted with a charge-coupled device camera (a); automatic measurement of contact angles (b).

Following testing, all specimens were examined under an optical microscope (SZH-131, Olympus, Tokyo, Japan) at a magnification of $\times 10$ and locations of bond failure were recorded. Types of failure were determined according to percentages of substrate-free material with adhesive failure, cohesive failure in dentin, and cohesive failure in resin or adhesive.

\section{Surface-free energy measurements}

Mandibular incisors were prepared as described in the preceding section and surface-free energy was determined by measuring surface contact angles for the three test liquids 1-bromonaphthalene, diiodomethane, and distilled water. Known surface-free energy parameters for test liquids are presented in Table 3. Surface-free energy values were determined according to contact angles in five specimens per treatment group for each dentin surface using a Drop Master DM500 instrument (Kyowa Interface Science, Saitama, Japan). The device was fitted with a charge-coupled device camera, which allowed automatic measurement of contact angles (Fig. 1). For each test liquid, equilibrium 
contact angles $(\theta)$ were measured using the sessiledrop method at $23 \pm 1^{\circ} \mathrm{C}$, and surface-free energy parameters of the solids were then determined based on fundamental concepts of wetting. Work of adhesion (W) between solids (S) and liquids (L) was determined using the Young-Dupré equation as follows:

$$
\mathrm{W}_{\mathrm{SL}}=\gamma_{\mathrm{L}}+\gamma_{\mathrm{S}}-\gamma_{\mathrm{SL}}=\gamma_{\mathrm{L}}(1+\cos \theta)
$$

where $\gamma_{\mathrm{SL}}$ is the interfacial free energy between the solid and liquid, $\gamma_{\mathrm{L}}$ is the surface-free energy of the liquid, and $\gamma_{\mathrm{S}}$ is the surface-free energy of the solid. After extending the Fowkes equation, the $\gamma_{\mathrm{SL}}$ can be expressed as follows:

$$
\begin{gathered}
\gamma_{S L}=\gamma_{L}+\gamma_{S}-2\left(\gamma_{L}{ }^{d} \gamma_{S}{ }^{d}\right)^{1 / 2}-2\left(\gamma_{L}{ }^{p} \gamma_{S}{ }^{p}\right)^{1 / 2}-2\left(\gamma_{L}{ }^{h} \gamma_{S}{ }^{h}\right)^{1 / 2} \\
\gamma_{L}=\gamma_{L d}+\gamma_{L p}+\gamma_{L h}, \gamma_{L}=\gamma_{S}{ }^{d}+\gamma_{S}{ }^{P}+\gamma_{S}{ }^{h}
\end{gathered}
$$

where $\gamma_{L}^{\mathrm{d}}, \gamma^{\mathrm{p}} \mathrm{L}$, and $\gamma_{\mathrm{L}}^{\mathrm{h}}$ are components of surfacefree energy $(\gamma)$ arising from dispersion forces, polar (permanent and induced) forces, and hydrogen-bonding forces, respectively. Surface-free energy $(\gamma)$ values were determined for the three test liquids, and surface energy parameters of the treated dentin surfaces were calculated based on these equations using add-on software and an interface measurement and analysis system (FAMAS, Kyowa Interface Science).

\section{Scanning electron microscopy (SEM)}

Treated dentin surfaces and resin-dentin interfaces were observed using SEM. In these experiments, airpowder polished dentin surfaces were rinsed with distilled water followed by air blowing. Ultrastructural observations of restorative/dentin interfaces were performed after embedding bonded specimens (stored at $37^{\circ} \mathrm{C}$ in distilled water for $24 \mathrm{~h}$ ) in epoxy resin (Epon 812, Nisshin EM, Tokyo, Japan), and incubating them at $37^{\circ} \mathrm{C}$ for an additional $12 \mathrm{~h}$. Embedded specimens were then sectioned through the diameter of the composite resin post, and surfaces of cut halves were polished using a grinder-polisher (Ecomet 4/Automet 2, Buehler) with \#600, \#1200, and then \#4000 grid SiC papers. Surfaces were finally polished on a special soft cloth using diamond paste (Buehler) with a grid size of 1.0 $\mu \mathrm{m}$. All SEM specimens were dehydrated in ascending concentrations of tert-butanol (50\% for $20 \mathrm{~min}$, $75 \%$ for $20 \mathrm{~min}, 95 \%$ for $20 \mathrm{~min}$, and $100 \%$ for $2 \mathrm{~h}$ ), and were then transferred to a critical-point dryer (Model ID-3, Eiko Engineering, Tokyo, Japan) for $30 \mathrm{~min}$. These surfaces were then subjected to argon-ion beam etching (Type EIS-200ER, Elionix, Tokyo, Japan) for $30 \mathrm{~s}$ with the ion beam (accelerating voltage, $1.0 \mathrm{kV}$; ion current density, $0.4 \mathrm{~mA} / \mathrm{cm}^{2}$ ) directed perpendicular to the polished surface. Surfaces were finally coated in a vacuum evaporator (Quick Coater Type SC-701, Sanyu Denshi, Tokyo, Japan) with a thin film of gold and were observed using a SEM instrument (ERA-8800FE, Elionix) at an accelerating voltage of $10 \mathrm{kV}$.

\section{Statistical analysis}

Data from SBS and surface-free energy tests were compared using one-way analysis of variance. Statistical analyses were performed using Sigma Plot software (Version 11.0, SPSS, Chicago, IL, USA).

\section{RESULTS}

Mean SBS to dentin ranged from 31.3 to $35.8 \mathrm{MPa}$ in the control group (without air-powder polishing) and were 27.2-32.9 $\mathrm{MPa}$ in the GL group, and 23.9$29.4 \mathrm{MPa}$ in the SB group (Table 4). Bond strengths following air-powder polishing were significantly lower under control conditions for all tested universal adhesives. However, adhesive failure predominated regardless of dentin pretreatments (Table 4).

Determinations of surface-free energies and their components (Table 5) showed decreased $\gamma$ s values with air-powder polishing, and were significantly lower for GL and SB groups compared with the control group.

\begin{tabular}{|c|c|c|c|}
\hline Adhesive & Control & GL & SB \\
\hline $\mathrm{SU}$ & $\begin{array}{c}35.6(4.1)^{\mathrm{a}} \\
{[0 / 0 / 10]}\end{array}$ & $\begin{array}{c}32.7(4.0)^{\mathrm{b}} \\
{[0 / 0 / 10]}\end{array}$ & $\begin{array}{c}27.5(3.7)^{\mathrm{c}} \\
{[0 / 0 / 10]}\end{array}$ \\
\hline GP & $\begin{array}{c}31.3(2.1)^{\mathrm{b}} \\
{[0 / 0 / 10]}\end{array}$ & $\begin{array}{c}27.2(2.8)^{\mathrm{c}} \\
{[0 / 0 / 10]}\end{array}$ & $\begin{array}{c}23.9(3.5)^{\mathrm{d}} \\
{[0 / 0 / 10]}\end{array}$ \\
\hline $\mathrm{AU}$ & $\begin{array}{c}35.8(3.1)^{\mathrm{a}} \\
{[0 / 0 / 10]}\end{array}$ & $\begin{array}{c}30.2(4.1)^{\mathrm{b}} \\
{[0 / 0 / 10]}\end{array}$ & $\begin{array}{c}26.7(4.1)^{\mathrm{c}} \\
{[0 / 0 / 10]}\end{array}$ \\
\hline $\mathrm{AB}$ & $\begin{array}{c}34.5(3.2)^{\mathrm{a}} \\
{[0 / 0 / 10]}\end{array}$ & $\begin{array}{c}32.9(3.8)^{\mathrm{b}} \\
{[0 / 0 / 10]}\end{array}$ & $\begin{array}{c}29.4(3.9)^{\mathrm{b}} \\
{[0 / 0 / 10]}\end{array}$ \\
\hline
\end{tabular}
Whereas estimated $\gamma \mathrm{s}^{\mathrm{d}}$ values remained relatively

Table 4 Influence of air-powder polishing on shear bond strengths of universal adhesive systems

Unit, MPa; values in parenthesis are standard deviations $(n=10)$

Values with the same superscript letters do not differ significantly $(p>0.05)$

Values in square parentheses indicate failure modes, (mixed failure/cohesive failure in dentin/adhesive failure) 
Table 5 Surface-free energy and component values for dentin-surface treatments

\begin{tabular}{|c|c|c|c|c|}
\hline & $\gamma \mathrm{s}$ & $\gamma S^{\mathrm{d}}$ & $\gamma \mathrm{s}^{\mathrm{p}}$ & $\gamma \mathrm{s}^{\mathrm{h}}$ \\
\hline Control & $53.4(1.6)$ & $36.4(1.6)$ & $0.0(0.0) \square$ & $17.0(2.3)$ \\
\hline GL & $47.2(2.6) \square$ & $36.3(1.9)$ & $0.0(0.0)$ & $10.9(1.7)$ \\
\hline SB & $46.7(2.5)-$ & $38.5(1.3)$ & $0.0(0.0)$ & $8.2(1.5)$ \\
\hline
\end{tabular}

Unit, $\mathrm{mN} / \mathrm{m}$; values in parenthesis are standard deviations $(n=5)$

Vertical lines between values indicate no significant differences $(p>0.05)$

$\gamma \mathrm{S}$ : surface-free energy, $\gamma \mathrm{s}^{\mathrm{d}}$ : dispersion force, $\gamma \mathrm{s}^{\mathrm{p}}$ : polarity force, $\gamma \mathrm{s}^{\mathrm{h}}$ : hydrogen bonding force
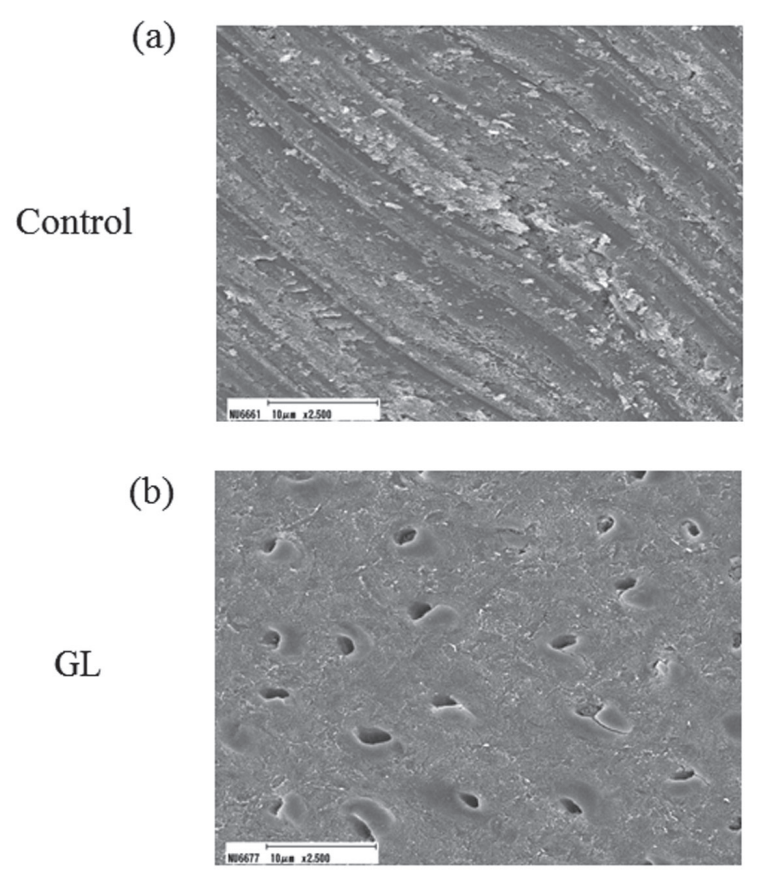

(c)

SB

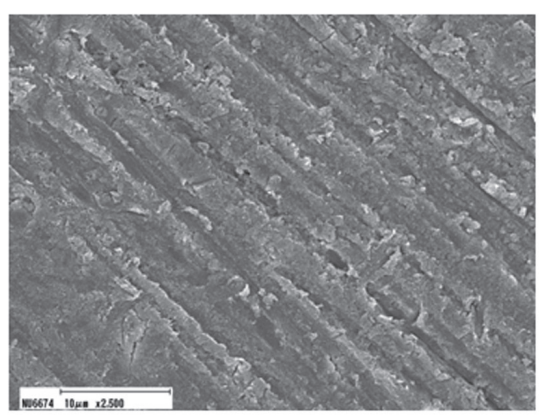

Fig. 2 Representative SEM photomicrographs of treated dentin surfaces; original magnification, 2,500×; (a) control, (b) glycine, and (c) sodium bicarbonate.

constant in the range of $36.3-38.5 \mathrm{mN} / \mathrm{m}$ for all surfaces, $\gamma \mathrm{s}^{\mathrm{h}}$ values in GL and SB groups were significant lower than those in the control group.

SEM observations of treated dentin surfaces (Fig. 2) revealed scratches created by $\mathrm{SiC}$ paper on dentin surfaces without air-powder polishing (control group), and smear layers over dentin tubules were observed. In contrast, smear layers were removed and dentin tubules were open in the GL group. However, after treatment with sodium bicarbonate powder, dentin surfaces were covered with smear layers and were rough.

SEM observations of resin/dentin interfaces (Fig. 3) showed excellent adaptation to the dentin surface at resin-dentin interfaces in all groups. Moreover, adhesive layers were approximately $5-10 \mu \mathrm{m}$ in thickness. However, resin tag formations in dental tubules were observed between dentin and adhesive in the GL group (Figs. 3c and d).

\section{DISCUSSION}

Due to their efficacy and convenience, air-powder polishing devices are increasingly used to remove biofilms, plaques, and stains from tooth surfaces ${ }^{9)}$. Accordingly, air-powder polishing reportedly removes dental debris more effectively than curets, ultrasonic scalers, and rubber cups ${ }^{2,8}$. However, some powders can cause damage to tooth surfaces during polishing. In a previous study, air-powder polishing of dentin surfaces using sodium bicarbonate powder increased damage to the tooth substructure and decreased bonding performance of two-step and single-step self-etching adhesive systems $\mathrm{s}^{4}$, leading to the development of a prophylactic polishing powder ${ }^{15,16)}$. Glycine is a highly water-soluble amino acid with a lower Morse hardness index than sodium bicarbonate, and has very low toxicity, is nonallergenic, has a light sweet taste, and is used as a food flavor enhancer, indicating high suitability for intra-oral use. Additionally, glycine powder particle sizes are approximately four times smaller than those of conventional sodium bicarbonate powder. However, it remains unclear whether air-powder polishing using glycine powder affects dentin adhesion of universal adhesives.

Highly effective bonding performance of universal adhesives reflects chemical bonding capacity of functional monomers to various substrates ${ }^{17-20)}$. Moreover, the extent to which liquid drops wet dentin surfaces depends on chemical interactions between the liquid and dentin, and on physical considerations such as capillary action, and surface roughness ${ }^{21)}$. 
SU

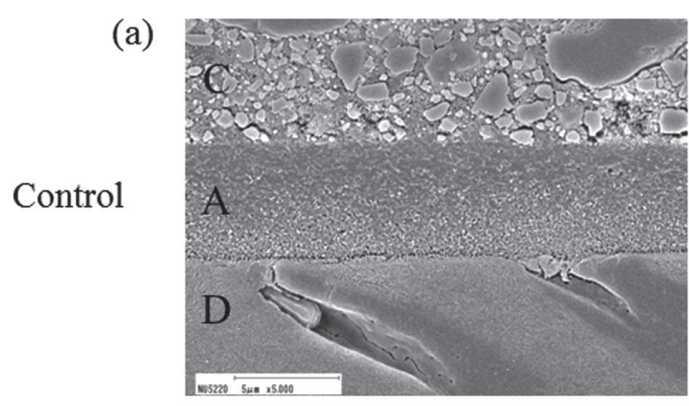

(c)

GL

SB

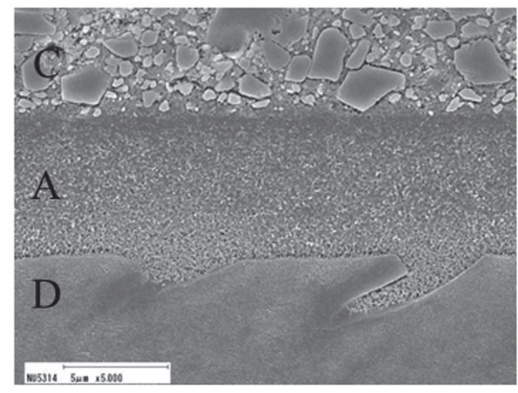

(e)

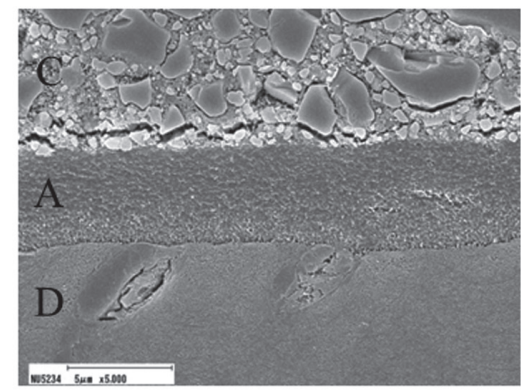

(b)

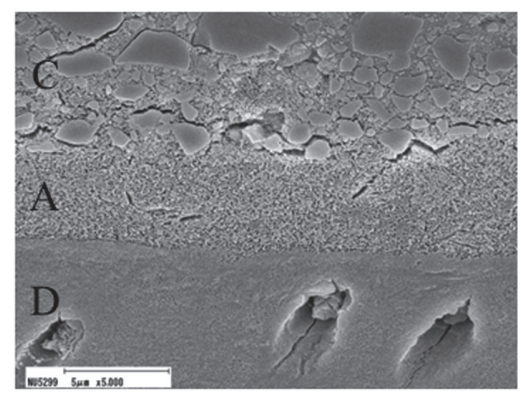

(d)

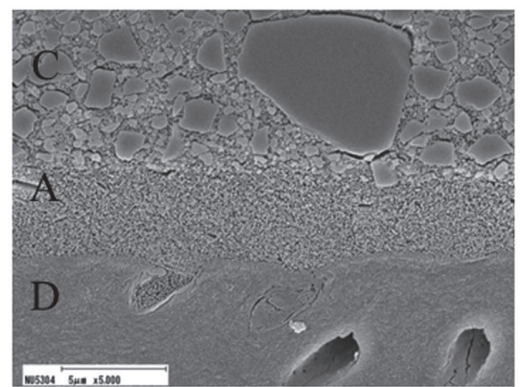

(f)

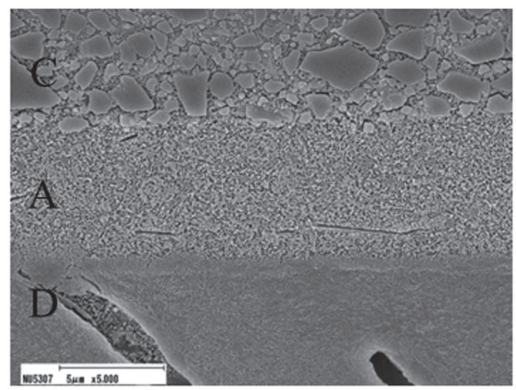

Fig. 3 Representative SEM photomicrographs of the dentin-resin interface; original magnification, 5,000×; (a) control with Scotchbond Universal Adhesive (SU), (b) control with G-Premio Bond (GP), (c) SU with glycine, (d) GP with glycine, (e) SU with sodium bicarbonate, (f) GP with sodium bicarbonate.

Investigations of air-powder polished dentin surfaces using measurements of surface-free energy may provide novel insights into bonding mechanisms between dentin and adhesive. Herein, we investigated the influence of air-powder polishing on the bonding performance of universal adhesives by measuring SBS and surface-free energies.

Compared with the control group, dentin bond strength of universal adhesives decreased significantly after air-powder polishing, suggesting that air-powder polishing influences bonding between dentin and adhesive. Subsequent SEM observations of dentin surfaces showed that smear layers remained on dentin surfaces in the SB group, suggesting that the presence of residual SB powder on dentin surfaces leads to chemical and/or mechanical changes of collagen fibrils, and prevention of adhesive penetration into dentin. Furthermore, the relatively higher $\mathrm{pH}$ of sodium bicarbonate $(\mathrm{pH}$ 8.3) may reduce the etching effect of universal adhesives, leading to reduced bonding performance ${ }^{22}$. In contrast, SEM analyses of dentin surfaces in the GL group showed that smear layers were removed, and indicated the presence of dentin tubules. These observations suggest that bonding resins penetration demineralized dentin better in the GL group than in SB and control groups (Figs. 3c and d).

Bonding between dentin and resin composites depends on several factors, including adhesion and wetting characteristics. Previous studies report assessments of wetting of adhered surfaces according to contact angles ${ }^{23,24)}$, and the surface-free energy of a solid $(\gamma \mathrm{s})$ reportedly reflects dispersion $\left(\gamma \mathrm{s}^{\mathrm{d}}\right)$, polarity $\left(\gamma \mathrm{s}^{\mathrm{p}}\right)$, and hydrogen-bonding $\left(\gamma \mathrm{s}^{\mathrm{h}}\right)$ forces ${ }^{25}$. Whereas dispersion forces represent London interactions between apolar molecules, polar (non-dispersion) forces include electrostatic, metallic, and dipolar interactions. In addition to these two parameters of $\gamma \mathrm{s}$, we determined hydrogen-bonding forces between water and hydroxyl 
groups. However, because hydration of adhered surfaces strongly influences wettability, accurate determinations of polar interactions and hydrogenbonding characteristics are warranted to assess interactions between adhesives and water ${ }^{26)}$. Thus, separate estimations of dipolar interactions and those of hydrogen-bonding interactions may provide novel insights into mechanisms of wettability and bonding characteristics of adhesives.

In the present study, $\gamma \mathrm{s}^{\mathrm{d}}$ values of dentin surfaces remained relatively constant (36.3-38.5 $\mathrm{mN} / \mathrm{m})$ compared with surface-free energy, regardless of the surface treatment. In a previous study, many surfaces had $\gamma \mathrm{s}^{\mathrm{d}}$ values that were close to $40 \mathrm{mN} / \mathrm{m}^{27)}$, and other studies attributed these values to adsorption of a socalled "atmospheric contamination layer" ${ }^{28)}$, particularly on chemically and/or mechanically activated surfaces ${ }^{29}$. Hence, the presence of this atmospheric layer may explain similarities of $\gamma \mathrm{s}^{\mathrm{d}}$ values between the present treatment groups. $\gamma \mathrm{s}^{\mathrm{p}}$ Values reflect polar interactions that are often referred to as non-dispersion forces, and are a measure of hydrophilic interactions with hydroxyapatite. The present $\gamma \mathrm{s}^{\mathrm{p}}$ values were low for all specimens, and the smear layers were predominantly hydrophobic. Moreover, demineralized dentin surfaces were covered by collagen fibrils following removal of smear layers and demineralization of superficial dentin, leading to lower polar surface. Thus, the presence of smear layers in control and SB groups, and collagen fibrils on dentin surfaces in the GL group, may have contributed to lower $\gamma \mathrm{S}^{\mathrm{p}}$ values. In contrast with these parameters, total surface-free energies and $\gamma \mathrm{s}^{\mathrm{h}}$ values of GL and SB groups were significantly lower than those in the control group. Hence, surface-free energy may reflect surface characteristics following air-powder polishing, and decreased $\gamma \mathrm{s}^{\mathrm{h}}$ values may indicate the presence of residual glycine and sodium bicarbonate powders on dentin surfaces and tubules. Furthermore, these data indicate reduced wettability against the adhesive, regardless of the presence of smear layers.

Finally, bond strengths of GP were significantly lower than for the other adhesives, regardless of airpowder polishing. Potentially, these observations reflect the presence of HEMA in the latter adhesives, which may promote expansion of collagen fibrils leading to enhanced adhesive monomer penetration into demineralized dentin ${ }^{30)}$. Taken together, the present data indicate significant differences in bond strength and surface-free energy following air-powder polishing.

\section{CONCLUSION}

The results of this study indicate that air-powder polishing with either glycine or sodium bicarbonate powder influences SBS and surface-free energies. However, glycine powder produced smaller changes in these surface parameters than sodium bicarbonate. Hence, clinical use of air-powder polishing requires consideration of the types of powder and universal adhesives.

\section{ACKNOWLEDGMENTS}

This work was supported in part by a Grand-in Aid Scientific Research (C) 26462896 and a Grand-in Aid Scientific Research (B) 15K20414 from the Japan Society for the Promotion of Science. This project was also supported, in part, by Sato Fund and by a grant from the Dental Research Center of the Nihon University School of Dentistry, Japan.

\section{REFERENCES}

1) Bühler J, Amato M, Weiger R, Walter C. A systematic review on the effects of air polishing devices on oral tissue. Int J Dent Hyg 2016; 14: 15-28.

2) Gutmann ME. Air polishing: a comprehensive review of the literature. J Dent Hyg 1998; 72: 47-56.

3) Duangthip D, Lussi A. Effects of application techniques and fissure types on the in vitro performance of two fissure sealants. Am J Dent 2004; 17: 137-142.

4) Frankenberger R, Lohbauer U, Tay FR, Teschner M, Nikolaenko SA. The effect of different air-polishing powders on dentin bonding. J Adhes Dent 2007; 9: 381-389.

5) Johnson WW, Barnes CM, Covey DA, Walker MP, Ross JA. The effects of a commercial aluminum airpolishing powder on dental restorative materials. J Prosthodont 2004; 13: 166 172 .

6) Nishimura K, Nikaido T, Foxton RM, Tagami J. Effect of airpowder polishing on dentin adhesion of self-etching primer bonding system. Dent Mater J 2005; 24: 59-65.

7) Sarah JG, Michelle LS, Jill LS. Air polishing: A review of current literature. J Dent Hyg 2013; 87: 173-180.

8) Petersilka GJ. Subgingival air-polishing in the treatment of periodontal biofilm infections. Periodontol 2000. 2011; 55: 124-142.

9) Pelka MA, Altmaier K, Petschelt A, Lohbauer U. The effect of air-polishing abrasives on wear of direct restoration materials and sealant. J Am Dent Assoc 2010; 141: 63-70.

10) Cardoso MV, de Almeida Neves A, Mine A, Coutinho E, Van Landuyt K, De Munck J, Van Meerbeek B. Current aspects on bonding effectiveness and stability in adhesive dentistry. Aust Dent J 2011; 56: 31-44.

11) de Gose MF, Shinohara MS, Freitas MS. Performance of a new one-step multi-mode adhesive on etched vs non-etched enamel on bond strength and interfacial morphology. J Adhes Dent 2014; 16: 243-250.

12) De Munck J, Van Meerbeek B, Satoshi I, Vargas M, Yoshida Y, Armstrong S, Lambrechts P, Vanherle G. Microtensile bond strengths of one- and two-step self-etch adhesives to bur-cut enamel and dentin. Am J Dent 2003; 16: 414-420.

13) Chaves P, Giannini M, Ambrosano GM. Influence of smear layer pretreatments on bond strength to dentin. J Adhes Dent 2002; 4: 191-196.

14) Chen C, Niu LN, Xie H, Zhou LQ, Jiao K, Chen JH, Pashley $\mathrm{DH}$, Tay FR. Bonding of universal adhesives to dentin - Old wine in new bottles? J Dent 2015; 43: 525-536.

15) Tada K, Kakuta K, Ogura H, Sato S. Effect of particle diameter on air polishing of dentin surfaces. Odontology 2010; 98: 3136.

16) Sahrmann P, Ronay V, Schmidlin PR, Attin T, Paqué F. Three-dimensional defect evaluation of air polishing on extracted human roots. J Periodontol 2014; 85: 1107-1114.

17) da Rosa WL, Piva E, da Silva AF. Bond strength of universal adhesives: A systematic review and meta-analysis. J Dent 2015; 43: 765-776.

18) Kim JH, Chae SY, Lee Y, Han GJ, Cho BH. Effects of multipurpose, universal adhesives on resin bonding to zirconia ceramic. Oper Dent 2015; 40: 55-62. 
19) Takamizawa T, Barkmeier WW, Tsujimoto A, Berry TP, Watanabe H, Erickson RL, Latta MA, Miyazaki M. Influence of different etching modes on bond strength and fatigue strength to dentin using universal adhesive systems. Dent Mater 2016; 32: e9-e21.

20) Suzuki T, Takamizawa T, Barkmeier WW, Tsujimoto A, Endo H, Erickson RL, Latta MA, Miyazaki M. Influence of etching mode on enamel bond durability of universal adhesive systems. Oper Dent 2016; 41: 520-530.

21) Inoue N, Tsujimoto A, Takimoto M, Ootsuka E, Endo $\mathrm{H}$, Takamizawa T, Miyazaki M. Surface free-energy measurements as indicators of the bonding characteristics of single-step self-etching adhesives. Eur J Oral Sci 2010; 118: 525-530.

22) Nikaido T, Kataumi M, Burrow MF, Inokoshi S, Yamada $\mathrm{T}$, Takatsu T. Bond strengths of resin to enamel and dentin treated with low-pressure air abrasion. Oper Dent 1996; 21: 218-224.

23) Aquilar-Mendoza JA, Rosales Leal JI, Rodriguez-Valverde MA, Gonzáles-López S, Cabrerizo-Vilchez MA. Wettability and bonding of self-etching dental adhesives. Influence of the smear layer. Dent Mater 2008; 24: 994-1000.
24) Bortolotto T, Ferrari M, Krejci I. Wetting ability of singlecomponent self-etching adhesives on enamel and dentin. Am J Dent 2008; 21: 59-63.

25) Hata T, Kitazaki Y, Saito T. Estimation of surface free energy of polymer solids. J Adhes 1987; 21: 177-194.

26) Gregoire G, Dabsie F, Dieng-Sarr F, Akon B, Sharrock P. Solvent composition of one-step self-etch adhesives and dentin wettability. J Dent 2011; 39: 30-39.

27) Tsujimoto A, Iwasa M, Shimamura $Y$, Murayama R, Takamizawa T, Miyazaki M. Enamel bonding of singlestep self-etch adhesives: Influence of surface-free energy characteristics. J Dent 2010; 38: 123-130.

28) Asmussen E, Peutzfeldt A, Sahafi A. Bonding of resin cements to post materials: influence of surface energy characteristics. J Adhes Dent 2005; 7: 231-234.

29) Combe EC, Owen BA, Hodges JS. A protocol for determining the surface free energy of dental materials. Dent Mater 2004; 20: $262-268$.

30) Takamizawa T, Barkmeier WW, Tsujimoto A, Scheidel DD, Erickson RL, Latta M, Miyazaki M. Effect of phosphoric acid pre-etching on fatigue limits of self-etching adhesives. Oper Dent 2015; 40: 379-395. 